\title{
Planarity as a driver of Spatial Network structure
}

\author{
Garvin Haslett and Markus Brede \\ Institute for Complex Systems Simulation, School of Electronics and Computer Science, \\ University of Southampton, Southampton SO17 1BJ, United Kingdom \\ g.a.haslett@ soton.ac.uk
}

\begin{abstract}
In this paper we introduce a new model of spatial network growth in which nodes are placed at randomly selected locations in space over time, forming new connections to old nodes subject to the constraint that edges do not cross. The resulting network has a power law degree distribution, high clustering and the small world property. We argue that these characteristics are a consequence of two features of our mechanism, growth and planarity conservation. We further propose that our model can be understood as a variant of random Apollonian growth. We then investigate the robustness of our findings by relaxing the planarity. Specifically, we allow edges to cross with a defined probability. Varying this probability demonstrates a smooth transition from a power law to an exponential degree distribution.
\end{abstract}

\section{Introduction}

In the last decade and a half tools from network science have been increasingly used to investigate a wide range of complex systems. Work within the discipline has sought to elucidate network structures not traditionally considered within the existing mathematical literature (Barabási and Albert, 1999; Watts and Strogatz, 1998; Brede, 2011) which are typically characterised by the power law degree distribution, high clustering and the small world property. Concomitantly systems as diverse as the protein network (Jeong et al., 2001), the Internet (Faloutsos et al., 1999), academic authorship (Newman, 2001) and the C. elegans neuronal network (Amaral et al., 2000; Haruna, 2011) have been examined to ascertain the extent to which they exhibit these properties. The insights gained from such studies benefit Alife practitioners in that they inform more nuanced representations of population structure in simulation (Buesser and Tomassini, 2012; Salathé et al., 2010; Lever et al., 2014).

More recently the sub-discipline of spatial networks has emerged as a subject in its own right (Barthélemy, 2011). The distinguishing feature here is that the nodes are ascribed a position in $\mathbb{R}^{2}$ and the distance between them are described, typically, by the Euclidean metric. The chief aim being to investigate the extent to which constraining connectivity in a manner related to node proximity influences system organisation. Application domains for these techniques include city science (Cardillo et al., 2006; Xie and Levinson, 2007; Jiang, 2007; Barthélemy and Flammini, 2008; Masucci et al., 2009; Chan et al., 2011; Courtat et al., 2011; Strano et al., 2012; Levinson, 2012; Rui et al., 2013; Gudmundsson and Mohajeri, 2013), electronic circuits (i Cancho et al., 2001; Bassett et al., 2010; Miralles et al., 2010; Tan et al., 2014), wireless networks (Huson and Sen, 1995; Lotker and Peleg, 2010), leaf venation (Corson, 2010; Katifori et al., 2010), navigability (Kleinberg, 2000; Lee and Holme, 2012; Huang et al., 2014) and transportation (Gastner and Newman, 2006; Louf et al., 2013).

In common with the parent discipline, a key aim of the spatial network literature is to establish appropriate null models for use as reference cases. A significant example amongst these is the random geometric graph which has proved useful to physicists working within continuum percolation (Balberg, 1985; Quintanilla et al., 2000) and has been the subject of extensive mathematical investigations (Dall and Christensen, 2002). A brief description of this model is that nodes are placed at random on the surface of a torus and pairs that lie within a specified maximum distance of each other are connected. Significantly, a power law degree distribution can only be observed in this model when the nodes themselves are are distributed inhomogeneously in space (Herrmann et al., 2003; Barnett et al., 2007; Bullock et al., 2010).

Other attempts have been made to discover spatial mechanisms which give rise to scale free degree distributions. A key review in this regard is that of Hayashi (Hayashi, 2006) which outlined three mechanisms: link length penalisation, space filling and embedding a scale-free network on a lattice. The first of these classes constrains the creation of a new edge based on some function of its length. The random geometric graph just mentioned is the fundamental example of these. Subsequent adaptions that demonstrate the power law property create connections based on a product of node weight and a power of the distance (Masuda et al., 2005; Manna and Sen, 2002).

The second class recursively partitions the space by adding new nodes to the plane and then connects them to 
the existing graph. One approach places the nodes randomly, selects the nearest edge and connect to one of its end nodes (Mukherjee and Manna, 2006). The authors posit that the power law ensues due to equivalence, in the limit of infinite system size, to a seminal model by Dorogovetsev et al. (Dorogovtsev et al., 2001). The second example in this class, Apollonian Networks (Andrade Jr et al., 2005; Doye and Massen, 2004), are a fractal construction that begin with a triangle in the plane which is then trisected to produce $K_{4}$. This step is repeated ad infinitum with all triangles present at each stage being further trisected.

The final class assigns an implicit degree $k$ to all the nodes in a lattice. Members of the lattice are then selected at random and connected to their $k$ nearest neighbours subject to a distance constraint (Ben-Avraham et al., 2003; Rozenfeld et al., 2002).

In the next section we present a model, named planar growth, which is a contribution to the space filling class. The model is so named because it is a growing process that only accepts new edges that do not cross each other in $\mathbb{R}^{2}$. Alongside it we also describe two models, no growth and no planarity that are are used as reference cases to illuminate the outcomes observed in the planar growth model. In the subsequent section we present our initial results from these models chief among them that a power law degree distribution is observed. We then produce a variation of the Random Apollonian Network which places the nodes uniformly in space. This new model is identical to planar growth while making clear a relationship with existing Apollonian literature. It also lends itself to more efficient realisation and we use it to produce networks of a greater scale. In the penultimate section we look at the consequences of relaxing the planarity constraint. In the final section we present our conclusions.

\section{Models}

Planar growth (PG) takes place upon a unit Euclidean square with rigid boundary conditions. We wish to begin the process with a small, i.e. statistically insignificant, population that has average degree $m$, the number of new connections that will be made for each new node when we begin the growth process proper. To do so we place ten nodes uniformly at random within the square. Each new node is connected to one of those already present; this target node being chosen so that the new edge does not cross an existing one.

Once all ten nodes have been placed, unconnected pairs are chosen randomly and edges added between them, again subject to the caveat that planarity is maintained. We continue until either $m \times 10$ edges are present or all possible pairs have been tried.

The algorithm now enters the growing phase where the following steps are repeated $n$ times, resulting in a network of size $n+10$ nodes and $m(n+10)$ edges:

\section{Step 1}

Place a new node uniformly at random within the square.

\section{Step 2}

Choose an existing node at random. If all existing nodes have been tried then reject the current node and return to step 1.

Step 3

Connect the new node and the existing node. If this connection results in crossed edges then reject it and repeat step 2, otherwise accept it and proceed.

\section{Step 4}

If the new node now has $m$ connections continue to step 5. Otherwise attempt to acquire another connection for the new node by returning to step 2 .

\section{Step 5}

If $n$ nodes have been added to the network then finish, otherwise return to step 1 .

The algorithm just outlined consists of two ingredients: (i) growth and (ii) planarity conservation. In the following section we make use of two further models, each containing just one of these two aspects. Then, in a manner following Barabási \& Albert (Barabási and Albert, 1999), we compare outcomes between the original and these simpler models thereby determining the extent to which growth and planarity contribute to the overall outcome.

The first of these, the no planarity model, is similar to PG in that it is a growth process on a unit square. It differs in that edge connections are always allowed. This scenario is indistinguishable from the uniform attachment model originally introduced by Barabási \& Albert which was shown to have an exponential degree distribution (Barabási et al., 1999).

The other, no growth, places $n$ nodes at random on the unit square. Pairs of these nodes are then chosen at random and connections formed between them. Planarity conservation is still enforced meaning that new edges are only permitted where they do not cross existing ones. This algorithm continues until $n \times m$ edges have been added to the network.

\section{Results}

In figure 1 we present three visualisations of small ( $n=$ 250) graphs produced by each of the mechanisms. Qualitatively, the no planar plot has the most unstructured appearance. By contrast the PG and the no growth scenarios both exhibit open regions that are not crossed by edges and a 'bunching' effect whereby edges connected to higher degree hubs are spread out in a fan like structure. It also appears that the hubs for the planar growth experiment are of a higher degree than those of the no growth model.

We investigate the node degree quantitatively by plotting the cumulative degree distribution of two PG experiments, of orders $n=10^{3}$ and $n=10^{4}$ and $m=2$, alongside a no planarity experiment of order $n=10^{4}$ and a no growth 


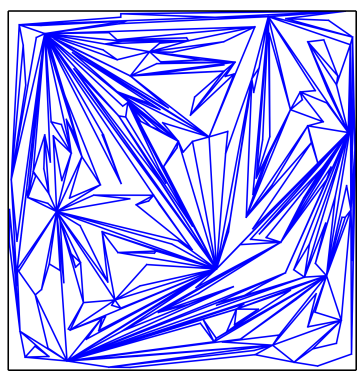

(a) Planar growth

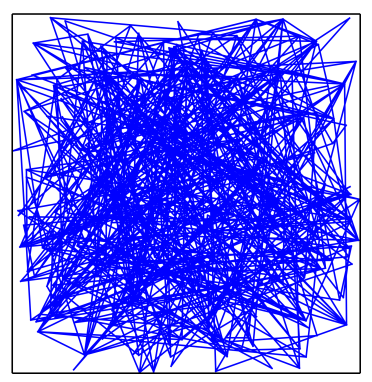

(b) No planar

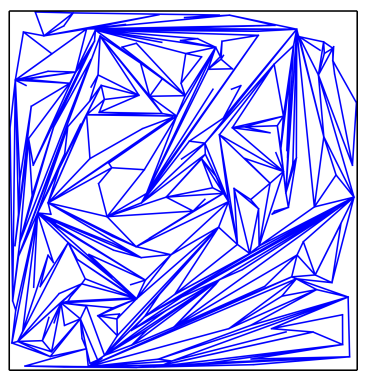

(c) No growth

Figure 1: Visualisation of planar growth, no planar and no growth networks with $n=250$ and $m=2$.

experiment of order $n=5 \times 10^{3}$ in figure 2 a. Each point is the average of the degree data from twenty experiments. Note that the restricted size of the no growth case is due to computational limits. These results show the degree distributions of both reference cases to be exponential. This is in contrast to the two planar growth cases; both of which seem to approximate a power law distribution, i.e. the probability, $p_{k}$ of observing degree $k$ is distributed as $p_{k} \sim k^{-\alpha}$. We have estimated the exponent in the $n=10^{4}, m=2$ case using the method of Maximum Likelihood Estimators outlined in Clauset et al. (Clauset et al., 2009) finding it to be $\alpha_{m=2}=2.84 \pm 0.01$.

We present further statistical evidence for the presence of power laws in the form of scaling of the maximum degree with system size for different network types. In figure $2 \mathrm{~b}$ we plot how the mean maximum degree observed during these experiments varies with the size of the network. Following Newman (Newman, 2003) we also plot $\left\langle k_{\max }\right\rangle \sim n^{1 /(\alpha-1)}$, where $\left\langle k_{\max }\right\rangle$ is the mean value of the maximum degree. This relationship is the analytically calculated value of $\left\langle k_{\max }\right\rangle$ under the assumption that $\mathrm{k}$ is distributed as a power law with exponent $\alpha$. Specifically, we have used the exponent $\alpha_{m=2}$ estimated above and figure $2 \mathrm{~b}$ demonstrates good agreement with the observations.

By standard techniques it can be shown that the expected value of the $i$ th member of a sequential ordering of the random variables of an exponential distribution with parameter $\lambda$ is $E\left[X_{i}\right]=H_{i} / \lambda$ where $H_{i}$ is the $i$ th harmonic number. We therefore expect, for a network with an exponential degree distribution, $\left\langle k_{\max }\right\rangle \sim H_{n}$, i.e. $\left\langle k_{\max }\right\rangle$ will grow logarithmically. The plots for the No Planarity and No Growth networks both conform to this prediction.

Taken together these plots indicate that the planar growth networks match expected behaviour for a power law degree distribution while, conversely, the two reference cases match that of an exponential one. However, note that in the planar growth cases the cumulative plot only extends over one order of magnitude along the $k$ axis, an outcome that needs confir- mation for larger system sizes. We will address this matter in the following section. Furthermore, the fact that the no growth and the no planarity models result in an exponential distribution leads us to conclude that neither growth nor planarity is sufficient to account for the scale free distribution; rather it is the combination of the two that is required. It is also interesting to note that although the no growth appeared visually similar to PG it is, in this regard, more similar to no planarity.

We now determine whether planar growth gives rise to the small-world property. Informally, small world networks have a high degree of community structure while maintaining a low average shortest path length. As such, they are believed to underpin the efficient transfer of information in large populations originally considered by Milgram (Milgram, 1967).

Community structure can be quantified by first introducing the clustering for an individual node $i$ :

$$
c_{i}=\frac{2 t_{i}}{k_{i}\left(k_{i}-1\right)}
$$

where $t_{i}$ is the number of triangles which have $i$ as a vertex and $k_{i}$ is the degree of node $i$. Therefore, $c_{i}=0$ when none of $i$ 's neighbours are connected and $c_{i}=1$ when they all are. Clustering $c$ for a whole network is then the average of $c_{i}$ over all the nodes in the network. Small worlds are then those networks that display high $c$ and a logarithmic increase in $l$, the average number of hops in the shortest path between pairs of nodes, with system size $n$ (Watts and Strogatz, 1998).

In a random network of size $n$ and average degree $m$ clustering follows $c_{\text {random }} \sim m / n$ and average shortest path length scales as $l_{\text {random }} \sim \ln (n) / \ln (m)$ (Watts and Strogatz, 1998); so for an $n=10^{4}, m=2$ random network this gives estimates of $c_{\text {random }}=0.0002$ and $l_{\text {random }}=13.29$. By comparison the observed values for a PG network using these values of $n$ and $m$ are $c_{m=2}=0.492 \pm 0.002$ and $l_{m=2}=6.6 \pm 0.1$, giving a first indication that planar net- 


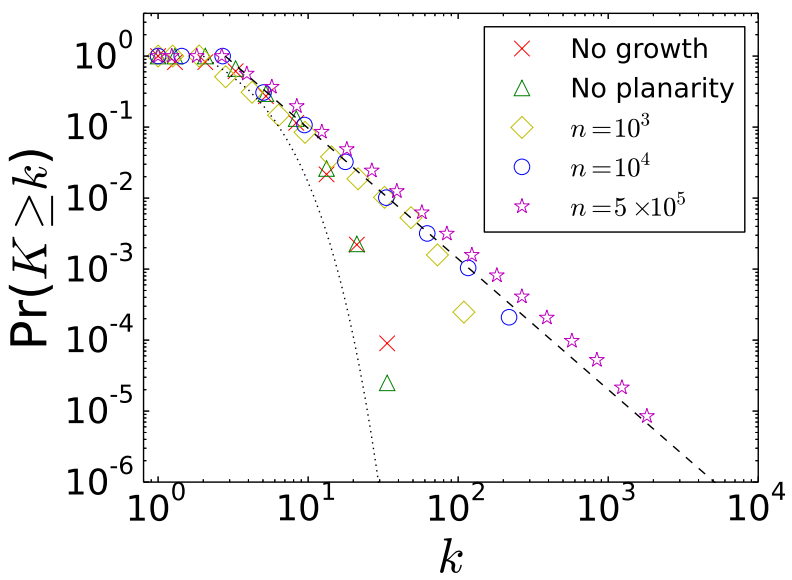

(a) Cumulative degree density

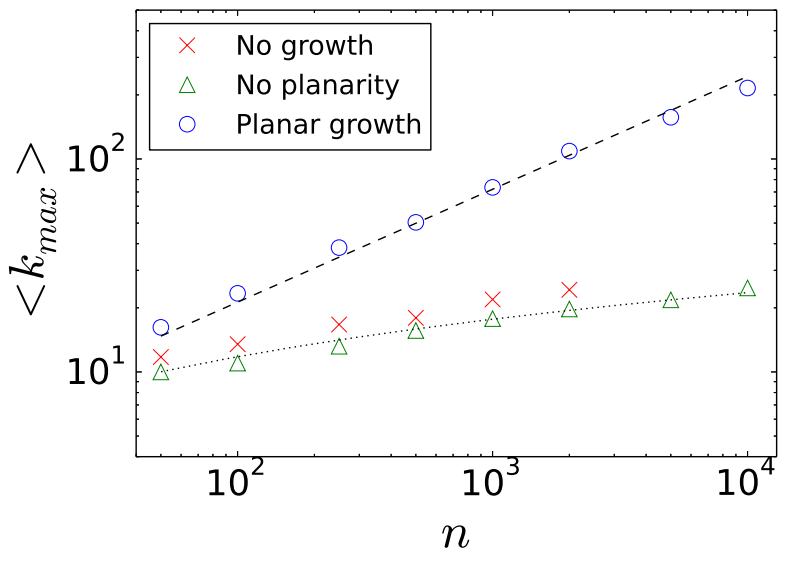

(b) Average maximum degree

Figure 2: Cumulative degree density for networks created using PG of orders $n=10^{3}$ and $10^{4}$, APG of order $5 \times 10^{5}$, No Planarity of order $n=10^{4}$ and No Growth of order $n=5 \times 10^{3}$. Dashed line is the best fit of APG experiment, a power law with exponent, $\alpha_{\mathrm{APG}}=2.84$. Dotted line is the degree distribution predicted by Barabási et al. (Barabási et al., 1999). (2a). Average maximum degree observed for PG with $m=2$, No Planarity and No Growth networks of varying order. Error bars representing standard deviation not shown but are similar in magnitude to the points. Dashed line is the expected value of the maximum degree for a power law with exponent $\alpha_{m=2}=2.84$, the estimated value of the exponent in the $n=10^{4}, m=2$ case. Dotted line is a plot of a logarithmic function. (2b).

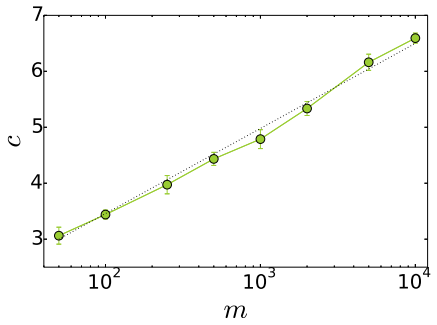

(a) Path length varying with $n$.

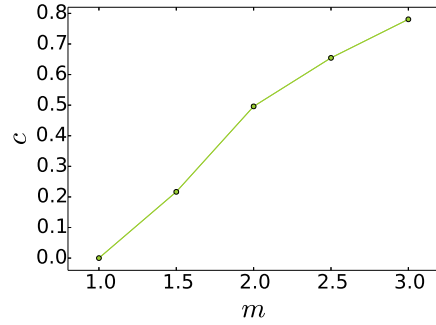

(b) Clustering varying with $m$.

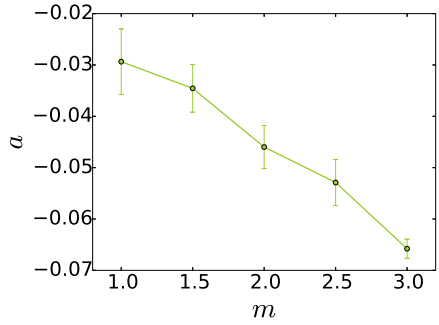

(c) Assortativity varying with $m$

Figure 3: Average shortest path length for $m=2$ networks with varying size. For each network size twenty experiments were performed. Dashed line is a logarithmic function. (3a). Average clustering observed in twenty PG networks with $n=10^{4}$ and varying $m$ (3b). Average assortativity observed in twenty PG networks with $n=10^{4}$ and varying $m$. (3c)

work growth indeed results in a small world. We further investigate the scaling of path length with system size in figure 3a where we plot $l$ against $n$ for a series of networks with $m=2$. The straight line on this plot indicates that $l$ is growing logarithmically with $n$ confirming the small world claim.

Figure $3 \mathrm{~b}$ plots how the clustering coefficient, $c$, varies with $m$ for networks of a constant size. What we see is clustering rising from $c_{m=1}=0.0$ to $c_{m=3}=0.7806 \pm 0.0003$. These high values of $c$ are the consequence of new nodes being placed within a $\mathrm{v}$-shape formed by three nodes and two edges. When this happens formation of a new triangle becomes likely since the edges constrain the possible connections the new node can make, in particular increasing the likelihood that it will connect to the apex of the $\mathrm{v}$ shape and one of its neighbours. It is interesting to note at this point that the clustering for the no growth experiment is $c_{N G}=0.29 \pm 0.02$ while that for a $n=5000, m=2$ network is $c_{n=5000}=0.497 \pm 0.006$ indicating that similar tendencies exist in the no growth case but not to the extent that they act in PG.

Finally for this section figure $3 \mathrm{c}$ plots how the assortativity varies with $m$ for PG networks of order $n=10^{4}$, showing it to decrease gradually from $a_{m=1}=-0.029 \pm 0.006$ to $a_{m=3}=-0.066 \pm 0.002$, i.e. the networks are mildly disassortative and this tendency increases as $\mathrm{m}$ increases. These results are in line with the well known fact that random scale-free networks are disassortative (Maslov et al., 2004; Park and Newman, 2003). A partial explanation of this phenomenon that has been offered is that there is a limited amount of possible edges that can lie between high degree hubs (Maslov et al., 2004). So, in general, a scale-free 
network must feature connections between high and low degree nodes.

\section{Apollonian Planar Growth}

We now address the issue of the small system sizes in the previous section. The computational problem is that repeatedly checking the existing network for edge crossings is time consuming so we therefore seek an algorithm that realises PG more efficiently. To do so, we modify an existing process of network growth; the Random Apollonian network (RAN) (Zhou et al., 2005). This construction begins with $K_{4}$ embedded on the plane and one of its three faces is chosen at random. A node is then placed within this face and connected to the three vertices, thereby replacing the existing face with three new ones. The process continues, choosing a face of the triangulation uniformly at random and trisecting it, ad infinitum.

The original RAN did not ascribe an explicit position to its nodes. This leads to an interpretation that each new node lies in the barycentre of its containing triangle. Furthermore, note that there is a similarity with preferential attachment here in that the likelihood of a node being selected to receive a new connection is directly proportional to the number of faces it is a vertex of; a quantity that is equal to the number of edges incident to the node. Two points should be made here, firstly, there is a densification effect as new nodes a more likely to form in the vicinity of existing ones. Secondly, the degree distribution can be calculated analytically and is a power law with exponent equal to 3 .

We modify the RAN to place nodes uniformly by adding two features. Firstly, we weight face selection by area, i.e. a face of area 0.5 units is twice as likely to be selected as one of 0.25 units. Secondly, when a new node is created, it is placed uniformly at random within the chosen face. This model is exactly planar growth on a triangle using degree of connection $m=3$ and in light of this fact we name it Apollonian planar growth (APG). Computationally, the enforced trisecting may be represented efficiently as a growing ternary tree which, in turn, can be exploited to grow networks that are an order of magnitude larger than those created with PG.

Returning to figure $2 \mathrm{a}$ we see this increase in size reflected in the plot of APG's degree distribution which extends over two orders of magnitude along the $k$ axis. This evidence, in combination with the fact that APG is a variation upon a process known to produce a scale free network leads us to conclude that PG itself results in a power law degree distribution.

\section{Planarity Relaxation}

The distinct differences between the PG and no planarity degree distributions lead us to consider how robust our process is. To investigate this we introduce a new parameter; $\chi \in[0,1]$, the crossing probability; to step 3 of the PG algorithm. We no longer reject crossings outright; instead, each time a crossing is encountered it is allowed with probability $\chi$. For example, suppose a new edge crosses three existing ones, then the check will be applied three times and if each one is passed then the new edge is permitted.

Networks with $n=10,000, m=2$ and varying values of $\chi$ between 0.0 and 1.0 were grown and investigated using similar tools to those in the previous section. The relevant degree distributions are shown in figure $4 \mathrm{a}$. For low values of $\chi$ the plots appear to be a reasonable fit for the power law observed in the case when $\chi=0.0$. As we increase $\chi$ the plots become more akin to the exponential curve that we expect for the $\chi=1.0$ case.

Figure $4 \mathrm{~b}$ shows the average maximum degree observed in these experiments. Those plots with low values of $\chi$ again seem to be a closer to the calculated value for $\chi=0.0$. Conversely, high values approach our estimate for the exponential case.

In figures $4 c$ and $4 d$ we examine how the assortativity and clustering vary with crossing probability. In the first case we see that $a$ rises from -0.05 for $\chi=0.0$ to 0.15 when $\chi=1.0$. The curve of the plot is smooth although its rate of change is markedly more pronounced beyond $\chi=0.4$. In the latter case the clustering, $c$ falls from 0.49 when $\chi=0.0$ to a negligible value when $\chi=1.0$. The curve here is roughly linear with a lower gradient at the ends.

In summary we note that both the clustering and assortativity change smoothly across the $\chi$ parameter. We also highlight the degree density and average maximum degree plots where the individual curves for each value of $\chi$ appear in an ordered fashion between the fits for the $\chi=0.0,1.0$ cases. In light of this evidence we conclude that allowing edge crossing modifies the network from one with the PG properties to a uniform attachment model in a smooth, graded fashion with no abrupt transitions.

The values observed for assortativity and clustering in the $\chi=1.0$ case are consistent with those obtained from a numerical simulation of a uniform attachment model. The negligible clustering is expected since the new edges are not subject to any pressure to attach to a node that would cause c to increase. As $\chi$ is decreased the effect outlined in the previous section takes hold and the clustering increases.

High assortativity in the uniform attachment model is due to an ageing effect, i.e. older edges will be both more likely to be connected to each other and to receive a higher amount of connections over the course of the simulation. Here, the effect of decreasing $\chi$ is to introduce more high degree hubs into the simulation thus driving the outcome towards the disassortativity outlined in the previous section.

\section{Conclusion}

In this paper we have outlined a minimal model of spatial network construction. It's principal features are stepwise 


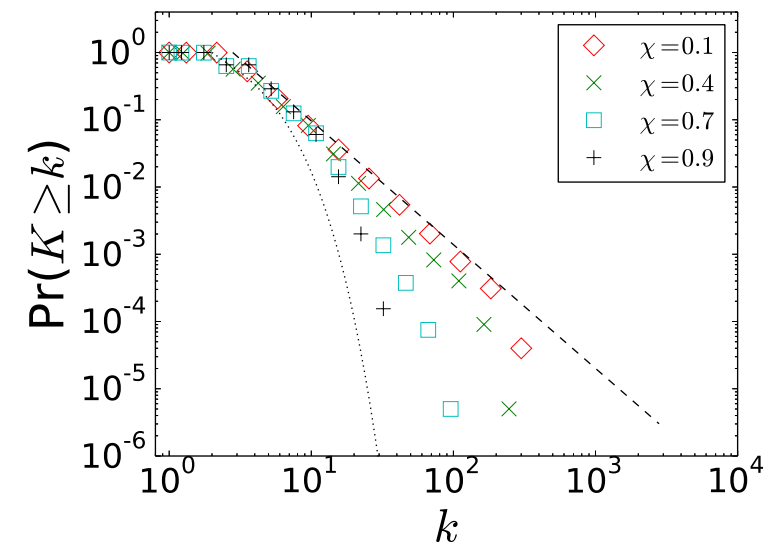

(a) Cumulative degree density.

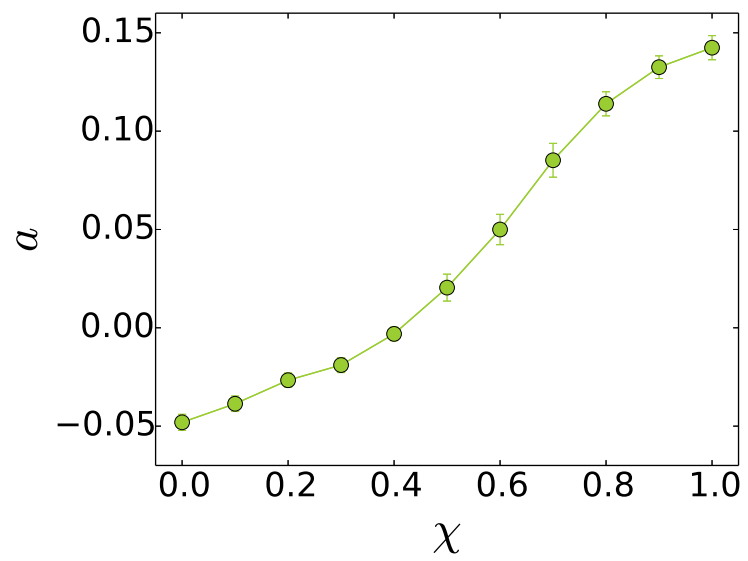

(c) Assortativity.

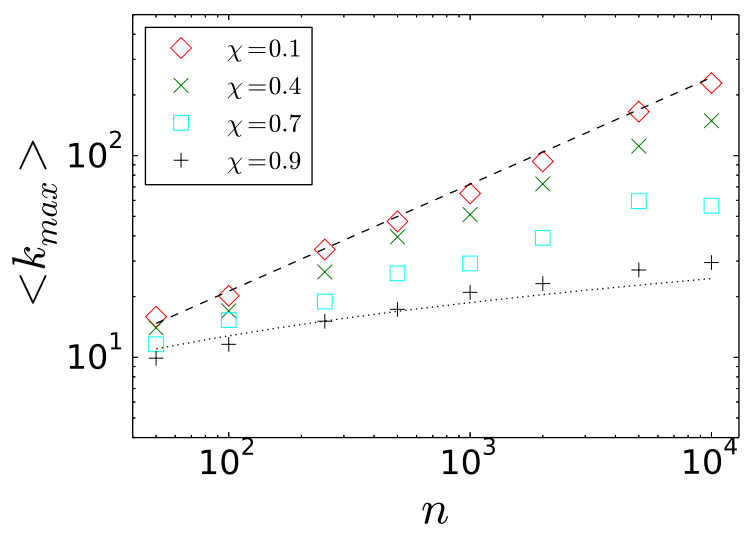

(b) Average maximum degree.

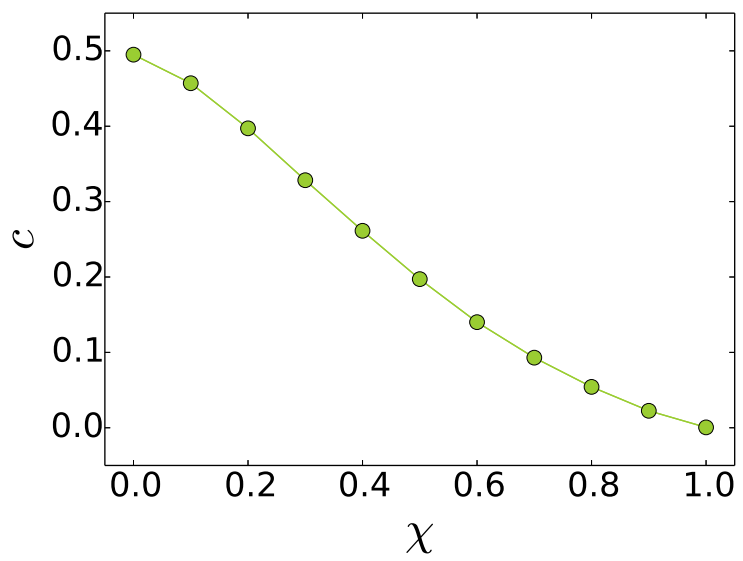

(d) Clustering.

Figure 4: Cumulative degree distribution for networks created using planar growth with the crossing probability modification. Dashed line is the power law with exponent $\alpha_{m=2}$, the best fit of the $\chi=0.0$ experiment (4a). Average maximum degree observed in the same experiments. Dashed and dotted lines are the same references plotted in figure $2 \mathrm{~b}$ and are fits for the $\chi=1.0$ and $\chi=0.0$ cases (4b). Average assortativity observed in PG networks with the crossing probability modification and varying $\chi(4 \mathrm{c})$. Average clustering (4d). 
growth and the conservation of planarity at each stage of that growth. We have produced a small world network with a power law degree distribution that exhibited disassortativity and high clustering. We have also demonstrated that individually neither the mechanism of growth nor that of planarity alone can fully account for these properties. In each of the cases where one of these ingredients was removed while retaining the other, a network with an exponential degree distribution was observed.

We further established a connection with an existing model of network growth, the Random Apollonian network. This was achieved by modifying the original RAN algorithm so that it became equivalent to the Planar Growth process. The result also lent further weight to the claim that the resulting network was scale free.

Empirical examples of spatial networks tend to exhibit a degree distribution that is peaked rather than scale free. Further, these networks tend to have low assortativity with a high clustering coefficient (Barthélemy, 2011). Moreover, there is a need for models that act as a reference case for these cases since, as yet, there is no general agreement within the literature on one to use.

In this light we have investigated the consequences of relaxing planarity by allowing edge crossing with varying probability. We found that key network measures transitioned from those associated with absolute enforcement of planarity to those associated with no planarity. We have described a continuum of networks, tunable by the crossing probability parameter which allow us to explore considerable variation in their structure. In this way the model can be tuned to act as a plausible baseline for comparison between theoretical models.

\section{References}

Amaral, L. A. N., Scala, A., Barthelemy, M., and Stanley, H. E. (2000). Classes of small-world networks. Proceedings of the National Academy of Sciences, 97(21):11149-11152.

Andrade Jr, J. S., Herrmann, H. J., Andrade, R. F., and da Silva, L. R. (2005). Apollonian networks: Simultaneously scalefree, small world, euclidean, space filling, and with matching graphs. Physical Review Letters, 94(1):018702.

Balberg, I. (1985). Universal percolation-threshold limits in the continuum. Physical review B, 31(6):4053.

Barabási, A.-L. and Albert, R. (1999). Emergence of scaling in random networks. Science, 286(5439):509-512.

Barabási, A.-L., Albert, R., and Jeong, H. (1999). Mean-field theory for scale-free random networks. Physica A: Statistical Mechanics and its Applications, 272(1):173-187.

Barnett, L., Di Paolo, E., and Bullock, S. (2007). Spatially embedded random networks. Physical Review E, 76(5):056115.

Barthélemy, M. (2011). Spatial networks. Physics Reports, 499(1):1-101.
Barthélemy, M. and Flammini, A. (2008). Modeling urban street patterns. Physical review letters, 100(13):138702.

Bassett, D. S., Greenfield, D. L., Meyer-Lindenberg, A., Weinberger, D. R., Moore, S. W., and Bullmore, E. T. (2010). Efficient physical embedding of topologically complex information processing networks in brains and computer circuits. PLoS Computational Biology, 6(4):e1000748.

Ben-Avraham, D., F Rozenfeld, A., Cohen, R., and Havlin, S. (2003). Geographical embedding of scale-free networks. Physica A: Statistical Mechanics and its Applications, 330(1):107-116.

Brede, M. (2011). Growth and optimality in network evolution. Artificial life, 17(4):281-291.

Buesser, P. and Tomassini, M. (2012). Evolution of cooperation on spatially embedded networks. Physical Review E, 86(6):066107.

Bullock, S., Barnett, L., and Di Paolo, E. A. (2010). Spatial embedding and the structure of complex networks. Complexity, 16(2):20-28.

Cardillo, A., Scellato, S., Latora, V., and Porta, S. (2006). Structural properties of planar graphs of urban street patterns. Physical Review E, 73(6):066107.

Chan, S. H. Y., Donner, R. V., and Lämmer, S. (2011). Urban road networks, spatial networks with universal geometric features? The European Physical Journal B-Condensed Matter and Complex Systems, 84(4):563-577.

Clauset, A., Shalizi, C. R., and Newman, M. E. (2009). Power-law distributions in empirical data. SIAM review, 51(4):661-703.

Corson, F. (2010). Fluctuations and redundancy in optimal transport networks. Physical Review Letters, 104(4):048703.

Courtat, T., Gloaguen, C., and Douady, S. (2011). Mathematics and morphogenesis of cities: A geometrical approach. Physical Review E, 83(3):036106.

Dall, J. and Christensen, M. (2002). Random geometric graphs. Physical Review E, 66(1):016121.

Dorogovtsev, S., Mendes, J., and Samukhin, A. (2001). Sizedependent degree distribution of a scale-free growing network. Physical Review E, 63(6):062101.

Doye, J. P. and Massen, C. P. (2004). Self-similar disk packings as model spatial scale-free networks. arXiv preprint condmat/0407779.

Faloutsos, M., Faloutsos, P., and Faloutsos, C. (1999). On powerlaw relationships of the internet topology. In ACM SIGCOMM Computer Communication Review, volume 29, pages 251-262. ACM.

Gastner, M. T. and Newman, M. E. (2006). Shape and efficiency in spatial distribution networks. Journal of Statistical Mechanics: Theory and Experiment, 2006(01):P01015.

Gudmundsson, A. and Mohajeri, N. (2013). Entropy and order in urban street networks. Scientific reports, 3 . 
Haruna, T. (2011). Global structure of directed networks emerging from a category theoretical formulation of the idea objects as processes, interactions as interfaces. In Advances in Artificial Life, ECAL 2011, Proceedings of the Eleventh European Conference on the Synthesis and Simulation of Living Systems, pages 310-317.

Hayashi, Y. (2006). A review of recent studies of geographical scale-free networks. Information and Media Technologies, 1(2):1136-1145.

Herrmann, C., Barthélemy, M., and Provero, P. (2003). Connectivity distribution of spatial networks. Physical Review E, 68(2):026128.

Huang, W., Chen, S., and Wang, W. (2014). Navigation in spatial networks: A survey. Physica A: Statistical Mechanics and its Applications, 393:132-154.

Huson, M. L. and Sen, A. (1995). Broadcast scheduling algorithms for radio networks. In Military Communications Conference, 1995. MILCOM'95, Conference Record, IEEE, volume 2, pages 647-651. IEEE.

i Cancho, R. F., Janssen, C., and Solé, R. V. (2001). Topology of technology graphs: Small world patterns in electronic circuits. Physical Review E, 64(4):046119.

Jeong, H., Mason, S. P., Barabási, A.-L., and Oltvai, Z. N. (2001). Lethality and centrality in protein networks. Nature, 411(6833):41-42.

Jiang, B. (2007). A topological pattern of urban street networks: universality and peculiarity. Physica A: Statistical Mechanics and its Applications, 384(2):647-655.

Katifori, E., Szöllősi, G. J., and Magnasco, M. O. (2010). Damage and fluctuations induce loops in optimal transport networks. Physical Review Letters, 104(4):048704.

Kleinberg, J. M. (2000). Navigation in a small world. Nature, 406(6798):845-845.

Lee, S. H. and Holme, P. (2012). Exploring maps with greedy navigators. Phys. Rev. Lett., 108:128701.

Lever, J. J., Nes, E. H., Scheffer, M., and Bascompte, J. (2014). The sudden collapse of pollinator communities. Ecology letters, 17(3):350-359.

Levinson, D. (2012). Network structure and city size. PloS One, 7(1):e29721.

Lotker, Z. and Peleg, D. (2010). Structure and algorithms in the sinr wireless model. ACM SIGACT News, 41(2):74-84.

Louf, R., Jensen, P., and Barthelemy, M. (2013). Emergence of hierarchy in cost-driven growth of spatial networks. Proceedings of the National Academy of Sciences, 110(22):88248829 .

Manna, S. S. and Sen, P. (2002). Modulated scale-free network in euclidean space. Physical Review E, 66(6):066114.

Maslov, S., Sneppen, K., and Zaliznyak, A. (2004). Detection of topological patterns in complex networks: correlation profile of the internet. Physica A: Statistical Mechanics and its Applications, 333:529-540.
Masucci, A., Smith, D., Crooks, A., and Batty, M. (2009). Random planar graphs and the London street network. The European Physical Journal B-Condensed Matter and Complex Systems, 71(2):259-271.

Masuda, N., Miwa, H., and Konno, N. (2005). Geographical threshold graphs with small-world and scale-free properties. Physical Review E, 71(3):036108.

Milgram, S. (1967). The small world problem. Psychology Today, 2(1):60-67.

Miralles, A., Comellas, F., Chen, L., and Zhang, Z. (2010). Planar unclustered scale-free graphs as models for technological and biological networks. Physica A: Statistical Mechanics and its Applications, 389(9):1955-1964.

Mukherjee, G. and Manna, S. (2006). Weighted scale-free networks in euclidean space using local selection rule. Physical Review E, 74(3):036111.

Newman, M. E. (2001). The structure of scientific collaboration networks. Proceedings of the National Academy of Sciences, 98(2):404-409.

Newman, M. E. (2003). The structure and function of complex networks. SIAM review, 45(2):167-256.

Park, J. and Newman, M. E. (2003). Origin of degree correlations in the internet and other networks. Physical Review E, 68(2):026112.

Quintanilla, J., Torquato, S., and Ziff, R. M. (2000). Efficient measurement of the percolation threshold for fully penetrable discs. Journal of Physics A: Mathematical and General, 33(42):L399.

Rozenfeld, A. F., Cohen, R., Ben-Avraham, D., and Havlin, S (2002). Scale-free networks on lattices. Physical Review Letters, 89(21):218701.

Rui, Y., Ban, Y., Wang, J., and Haas, J. (2013). Exploring the patterns and evolution of self-organized urban street networks through modeling. The European Physical Journal B, 86(3):1-8.

Salathé, M., Kazandjieva, M., Lee, J. W., Levis, P., Feldman, M. W., and Jones, J. H. (2010). A high-resolution human contact network for infectious disease transmission. Proceedings of the National Academy of Sciences, 107(51):22020-22025.

Strano, E., Nicosia, V., Latora, V., Porta, S., and Barthélemy, M. (2012). Elementary processes governing the evolution of road networks. Scientific Reports, 2.

Tan, H., Peng, M., Tse, C. K., and Wu, F. (2014). Global similarity tests of physical designs of circuits: A complex network ap proach. Applied Mathematics and Computation, 230:96-103.

Watts, D. J. and Strogatz, S. H. (1998). Collective dynamics of small-worldnetworks. Nature, 393(6684):440-442.

Xie, F. and Levinson, D. (2007). Measuring the structure of road networks. Geographical Analysis, 39(3):336-356.

Zhou, T., Yan, G., and Wang, B.-H. (2005). Maximal planar networks with large clustering coefficient and power-law degree distribution. Physical Review E, 71(4):046141. 